\title{
GROUP RINGS WHOSE TORSION UNITS FORM A SUBGROUP*
}

\author{
by SÔNIA P. COELHO and C. POLCINO MILIES
}

(Received 19th February 1992)

\begin{abstract}
In this note, we determine fields $K$ and groups $G$ that are either nilpotent or FC and such that the set of torsion elements of the group ring $K G$ forms a subgroup.
\end{abstract}

1991 Mathematics subject classification: $20 \mathrm{C} 07$.

\section{Introduction}

Let $U(K G)$ denote the group of units of the group ring of a given group $G$ over a field $K$. Also, we shall denote by $T=T(G)$ and $T U(K G)$ the set of elements of finite order in $G$ and $U(K G)$ respectively.

In this note, we shall consider groups $G$ that are either nilpotent or FC and determine conditions on $G$ and $K$ for $T U(K G)$ to be closed under multiplication, i.e. to be a subgroup of $U(K G)$. This question was first studied in [4] but the answer was incomplete because it depended on the fact that every idempotent of $K T$ is central in $K G$, a condition not fully understood at that time. Using the results in $[1,2]$, we are able to give a complete answer to this question. In particular, we do not need a technical hypothesis assumed in [4, Theorem 4.4] and we correct a gap in [4, Theorem 5.2]. In what follows, if a ring $R$ is such that its torsion units form a subgroup, we shall say, briefly, that $R$ has the t.p.p. (torsion product property).

\section{Group rings in characteristic $p>0$}

We remark first that, if $G$ is either a nilpotent or FC group, then $T$ is locally finite and that if $G \neq T$, then $G$ contains a central element of infinite order (see $[5,5.2 .22$ and 14.5.6]). We denote the Jacobson radical of a given ring $R$ by $J(R)$.

Lemma 2.1. Let $G$ be a group such that $T=T(G)$ is locally finite, and assume that either $G$ contains a central element of infinite order or $K$ is not algebraic over its prime field $\mathscr{P}(K)$. If $T U(K G)$ is a subgroup then, for every finite subgroup $T_{1} \subset T$, the quotient ring $K T_{1} / J\left(K T_{1}\right)$ is a direct sum of fields.

*Both authors were partially supported by a research grant from CNPq. 
Proof. Let $x$ denote be a central element of infinite order in $G$. Denote by $K T_{1}[x]$ the smallest subring of $K G$ containing $K T_{1}$ and $\{x\}$ and let $\phi:\left(K T_{1}\right)[x] \rightarrow$ $\left(K T_{1} / J\left(K T_{1}\right)\right)[x]$ the epimorphism induced by the natural map $K T_{1} \rightarrow K T_{1} / J\left(K T_{1}\right)$. Since $J\left(K T_{1}\right)$ is nilpotent and $x$ is central, it follows that $\operatorname{Ker}(\phi)=J\left(K T_{1}\right)[x]$ is a nilpotent ideal. Hence, $\phi$ induces, by restriction, epimorphisms of the respective unit groups and also of the respective sets of torsion elements. Then, it is easily seen that $\left(K T_{1} / J\left(K T_{1}\right)\right)[x]$ also has the t.p.p.

Since $K T_{1} / J\left(K T_{1}\right)$ is semisimple artinian, we have that

$$
\frac{K T_{1}}{J\left(K T_{1}\right)} \cong \bigoplus_{i=1}^{t} M_{n_{i}}\left(D_{i}\right)
$$

where $D_{i}$ is a division ring containing $K, 1 \leqq i \leqq t$.

For each index $i$ we have:

$$
\begin{aligned}
\left(M_{n_{i}}\left(D_{i}\right)\right)[x] & \cong\left(M_{n_{i}}\left(D_{i}\right) \otimes_{K} K\right)[x] \\
& \cong\left(D_{i} \otimes_{K} M_{n_{i}}(K) \otimes K\right)[x] \cong D_{i} \otimes_{K} M_{n_{i}}(K[x])
\end{aligned}
$$

Then, also $M_{n i}(K[x])$ has the t.p.p. It follows from [4, Prop. 2.2] that $n_{i}=1$. Then:

$$
\frac{K T_{1}}{\mathrm{~J}\left(K T_{1}\right)} \cong \bigoplus_{i=1}^{t} D_{i}
$$

Given any two elements $x, y \in T_{1}$ we have that $\bar{x}, \bar{y} \in \bigoplus_{i=1}^{i} T U\left(D_{i}\right)$ and [4, Prop. 2.1] shows they are central. Hence, $K T_{1} / J\left(K T_{1}\right)$ is commutative and the result follows.

A similar argument proves the statement in the case where $K$ contains an element $x$ which is transcendental over $\mathscr{P}(K)$.

Lemma 2.2. Let $K$ and $G$ be as in the previous lemma. If $T U(K G)$ is a subgroup, then $P$, the set of p-elements in $G$, is a normal subgroup of $G$ and $T^{\prime} \subset P$.

Proof. Assume that $\alpha$ is a p-element. Then, for some integer $n \geqq 1$ we have that $(\alpha-1)^{p^{n}}=\alpha^{p^{n}}-1=0$ i.e. $\alpha-1$ is a nilpotent element. We set $T_{1}=\langle\operatorname{supp}(\alpha)\rangle$. Then $T_{1}$ is finite and the image $\overline{\alpha-1}$ in $K T_{1} / J\left(K T_{1}\right)$ is also nilpotent. Then, Lemma 2.1 shows that $\alpha \in 1+J\left(K T_{1}\right)$.

Given two p-elements $\alpha, \beta \in G$, put $T_{1}=\langle\operatorname{supp}(\alpha), \operatorname{supp}(\beta)\rangle$; then we have that $\alpha \beta \in 1+$ $J\left(K T_{1}\right)$, which is a p-group.

Given $x, y \in T$, Lemma 2.1 shows that $K\langle x, y\rangle / J(K\langle x, y\rangle) \cong \bigoplus_{i} D_{i}$, a direct sum of fields; hence, $(x, y)-1=x y x^{-1} y^{-1}-1 \in J(K\langle x, y\rangle)$. Thus, there exists an integer $n \geqq 1$ such that $(x, y)^{p^{n}}=1$. Consequently $T^{\prime} \subset P$.

In what follows, we shall denote by $\Delta(G: P)$ the kernel of the natural homomorphism $K G \rightarrow K(G / P)$. 
Theorem 2.3. Let $G$ be a nilpotent or $F C$ group and let $K$ be a field with char $(K)=p>0$. Then $T U(K G)$ is a subgroup if and only if one of the following conditions holds:

(i) $G$ is abelian.

(ii) $G=T$ and $K$ is algebraic over its prime field $\mathscr{P}(K)$.

(iii) The set $P$ of p-elements in $G$ is a subgroup, $T^{\prime} \subset P$ and if $T / P$ is non central in $G / P$ then $\Omega$, the algebraic closure of $\mathscr{P}(K)$ in $K$, is finite and, for all $x \in G$ and all $p^{\prime}-$ elements $a \in T$, we have that $x_{a x}^{-1}$ is of the form $x a x^{-1}=a^{p^{r}} y$, where $r \geqq 0$ and $y \in P$. Furthermore, for every such an exponent $r$ we have that $[\Omega: \mathscr{P}(K)] \mid r$.

Proof. Assume that $T U(K G)$ is a subgroup, that $G$ is not abelian and that either $G \neq T$ or $K$ is not algebraic over $\mathscr{P}(K)$. From Lemma 2.2 we see that $P$ is a subgroup and that $T^{\prime} \subset P$.

Since $\Delta(G: P)$ is a locally nilpotent ideal, it follows that $K(G / P)$ also has the t.p.p. Since $T / P$ contains no p-elements, [7, Lemma VI.3.12] shows that if there exists a non central idempotent $e \in K(T / P)$, then $U(K(G / P))$ contains a subgroup which is isomorphic to $G L(m, K)$ with $m>1$. If $K$ is not algebraic over $\mathscr{P}(K)$ this yields a contradiction. On the other hand, if $G \neq T$, then [4, Theorem 4.1] shows directly that every idempotent of $K(T / P)$ is central in $K(G / P)$.

In both cases, [1] shows that (iii) holds.

To prove sufficiency, we observe that both (i) and (ii) imply readily that $K G$ has the t.p.p. Thus, assume that (iii) holds. Then, [1] shows that every idempotent of $K(T / P)$ is central in $K(G / P)$ and, as in [4, Theorem 4.4] we see that $K G$ has the t.p.p. also in this case.

\section{Group rings in characteristic 0}

Our first result holds in a slightly more general setting.

Lemma 3.1. Let $G$ be a group such that $T(G)$ is locally finite and let $K$ be a field of characteristic 0 . If $T U(K G)$ is a subgroup, then $T$ is abelian.

Proof. To prove our statement, we can assume that $T$ is finite. Then, we can write $K T \cong \bigoplus_{i=1}^{i} M_{n_{1}}\left(D_{i}\right)$. Since $M_{2}(\mathbf{Q})$ does not have the t.p.p. (see, for example [6, p. 20]), it follows immediately that $n_{i}=1,1 \leqq i \leqq t$.

Thus, $K T \cong \bigoplus_{i=1}^{t} D_{i}$ contains no nilpotent elements, so [7, Theorem VI.1.11] shows that $T_{1}$ is either abelian or a Hamiltonian group. Finally, if $T_{1}$ is Hamiltonian, it contains a subgroup of the form

$$
\mathscr{Q}=\left\langle a, b \mid a^{4}=1, a^{2}=b^{2}, b a b^{3}=a^{3}\right\rangle .
$$

Let $p$ be any prime and denote by $Z_{(p)}$ the localization of $\mathbf{Z}$ at the prime ideal $(p)$. It was shown in [3, Theorem 2] that $\alpha=x+y a$ with $x, y \in \mathbf{Z}, p \nmid x, p \mid y$, is a unit in $\mathbf{Z}_{(p)} \mathscr{Q}$, 
and therefore in $\mathbf{Q} G$ and that $b\left(b^{-1}\right)^{\alpha}=(b, \alpha)$ is not an element of finite order. Hence, $T_{1}$ must be abelian.

We can now correct [4, Theorem 5.2], which should be stated as follows.

Theorem 3.2. Let $G$ be a nilpotent or FC group and let $K$ be a field of characteristic 0 . Then, $T U(K G)$ is a subgroup if and only if the following conditions hold:

(i) $T$ is abelian.

(ii) For each $t \in T$ and each $x \in G$ there exists a positive integer $i$ such that $x t x^{-1}=t^{i}$ and, for each non central element $t \in T, K$ contains no root of unity of order $o(t)$.

Proof. Assume that $T U(K G)$ is a subgroup. We know, from the lemma above, that $T$ is abelian.

Also, every idempotent in $K T$ is central in $K G$, since, if this is not the case, as before, [7, Lemma VI.3.12] shows that $U(K G)$ contains a copy of $G L(m, K)$, with $m>1$. This yields a contradiction, because $M_{2}(\mathbf{Q})$ does not have the t.p.p.

Now, [2] shows that, for each $t \in T$ and each $x \in G$ we have that $x t x^{-1}=t^{i}$, as stated, and that for every non central element $t \in T, K$ contains no root of unity of order $o(t)$.

To prove sufficiency, notice that we may suppose that $G$ is finitely generated and, therefore, that $T$ is finite. Thus $K T=\bigoplus_{i=1}^{i} K_{i}$, a direct sum of fields. Let $S$ be a transversal of $T$ in $G$. Then, we know from [7, Lemma VI.3.22] that every unit $u \in K G$ can be written in the form $u=\sum_{i} f_{i} g_{i}$ where $0 \neq f_{i} \in K_{i}, g_{i} \in S, 1 \leqq i \leqq t$.

Since [2] show that the conditions in the statement of our theorem imply that every idempotent of $K T$ is central in $K G$, we have that $g_{i} f_{i}=f_{i}^{\prime} g_{i}$, for some $f_{i}^{\prime} \in K, 1 \leqq i \leqq t$. Hence:

$$
u^{m}=\sum_{i=1}^{t} f_{i} g_{i}^{m}
$$

where $\bar{f}_{i} \in K_{i}, 1 \leqq i \leqq t$. Thus, $u \in T U(K G)$ if and only if there exists an integer $m$ such that $g_{i}^{m}=1,1 \leqq i \leqq t$, i.e. if and only if $u \in U(K T)$. Since $T$ is abelian, it follows easily that $K G$ has the t.p.p.

\section{REFERENCES}

1. S. P. Coelro, A note on central idempotents in group rings, Proc. Edinburgh Math. Soc. 30 (1987), 69-72.

2. S. P. Coelho and C. Polcino Milies, A note on central idempotents in group rings II, Proc. Edinburgh Math. Soc. 31 (1988), 211-215.

3. M. M. Parmenter and C. Polcino Milies, Group rings whose torsion units form a nilpotent or FC group, Proc. Amer. Math. Soc. 68 (1978), 247-248.

4. C. Polcino Milies, Group rings whose torsion units form a subgroup II, Comm. Algebra 9 (1981), 699-712. 
5. D. J. S. Robinson, $A$ Course in the Theory of Groups (Springer-Verlag, New York, 1982).

6. J. J. Rotman, The Theory of Groups: An Introduction, 2nd ed. (Allyn and Bacon, Boston, 1973).

7. S. K. SehGal, Topics in Group Rings (Marcel Dekker, New York, 1978).

Instituto de Matemática e Estatistica

Universidade de Sáo Paulo

Caixa Postal 20570-Ag. Iguatem

01452-990-Sáo Paulo-Brasil. 